Louisiana State University

LSU Digital Commons

Faculty Publications

Department of Physics \& Astronomy

6-1-2011

\title{
Assessing the risk of second malignancies after modern radiotherapy
}

Wayne D. Newhauser

University of Texas MD Anderson Cancer Center

Marco Durante

Technische Universität Darmstadt

Follow this and additional works at: https://digitalcommons.Isu.edu/physics_astronomy_pubs

\section{Recommended Citation}

Newhauser, W., \& Durante, M. (2011). Assessing the risk of second malignancies after modern radiotherapy. Nature Reviews Cancer, 11 (6), 438-448. https://doi.org/10.1038/nrc3069

This Article is brought to you for free and open access by the Department of Physics \& Astronomy at LSU Digital Commons. It has been accepted for inclusion in Faculty Publications by an authorized administrator of LSU Digital Commons. For more information, please contact ir@lsu.edu. 


\title{
Assessing the risk of second malignancies after modern radiotherapy
}

\author{
Wayne D. Newhauser ${ }^{\star}$ and Marco Durante ${ }^{\ddagger}$ \\ "MD Anderson Cancer Center Radiation Oncology, 1515 Holcombe Boulevard Houston, Texas \\ 77030-4009, USA. \\ ‡GSI Helmholtz Center for Heavy-Ion Research, Department of Biophysics, Planckstrasse 1, and \\ Darmstadt University of Technology, Darmstadt 64291, Germany.
}

\begin{abstract}
Recent advances in radiotherapy have enabled the use of different types of particles, such as protons and heavy ions, as well as refinements to the treatment of tumours with standard sources (photons). However, the risk of second cancers arising in long-term survivors continues to be a problem. The long-term risks from treatments such as particle therapy have not yet been determined and are unlikely to become apparent for many years. Therefore, there is a need to develop risk assessments based on our current knowledge of radiation-induced carcinogenesis.
\end{abstract}

\begin{abstract}
Advances in paediatric cancer care have yielded steady gains in survival time over the past four decades. Today, in the United States, approximately $80 \%$ of children with cancer will survive 5 years or longer, compared with 30\% in 1960 (REF. 1). Epidemiological studies of survivors of childhood cancer revealed startling rates of disease and treatment-related health problems $^{2}$. Survivors experience an alarmingly high incidence of chronic health problems after their treatment ${ }^{3}$, including second primary cancers, cardiac toxicity and fertility problems, to name just a few ${ }^{4}$. This means that survivors experience more than an extra decade of ill health compared with children who have not been treated for cancer. Long-term survivors of childhood cancer who received radiotherapy are at a significantly increased risk for the development of second malignant neoplasms (SMNs) ${ }^{2}$ : the incidence of SMNs is around $10-20 \% 30$ years after treatment ${ }^{1}$. Indeed, the 5-year survival rate for children with medulloblastoma is $73 \%{ }^{1}$, but the 30 -year cumulative incidence of SMNs is $31 \%{ }^{2}$.

Reducing the risk of second cancers in survivors of childhood cancer will require the identification of patients at the greatest risk for $\mathrm{SMNs}^{5,6}$, improved treatment regimens ${ }^{7}$ and improved health care for adult survivors within the economic constraints that are placed on the health care system ${ }^{8}$. Research into cancer survival aims to better understand, prevent and
\end{abstract}

\footnotetext{
(C) 2011 Macmillan Publishers Limited. All rights reserved wnewhaus@mdanderson.org; M.Durante@gsi.de

Competing interests statement The authors declare no competing financial interests.

FURTHER INFORMATION Marco Durante's homepage: http://www.fkp.tu-darmstadt.de/groups/ag_durante/dur/index.en.jsp Wayne D. Newhauser's homepage: http://www.uthouston.edu/gsbs/faculty/faculty-directory/faculty-profiles.htm?id=1346643 GSI: http://www.gsi.de/forschung/bio/ ALL LINKS ARE ACTIVE IN THE ONLINE PDF
} 
treat late-occurring effects in long-term survivors while preserving the high rates of local control and long-term survival that are achieved with contemporary treatments ${ }^{7}$.

The path forwards presents challenges to researchers, policy makers and clinicians. Various approaches have been proposed to reduce the incidence of SMNs from radiotherapy, mainly by reducing radiation doses to healthy tissues by using new techniques and various types of radiation, such as protons or heavy ions. However, few clinical comparative effectiveness studies have been conducted ${ }^{9}$, and no long-term epidemiological studies of SMN incidence following proton or carbon therapies yet exist. Significant outcome-based evidence on SMN risk will not be forthcoming for many years as SMNs generally take a long time to develop. Moreover, it is currently unclear what these outcome data are likely to show or whether they will have much effect on the decision to use particle therapy as a mode of cancer treatment, as refinements are likely to be ongoing in this rapidly developing technological area. Therefore, decisions will have to be made using alternative types of evidence, as is customary and common in many other rapidly advancing disciplines of medicine. The evidence will by necessity comprise theoretical predictions.

There are compelling theoretical advantages for particle therapy ${ }^{10,11}$ compared with conventional X-ray treatment. The advantage of charged particles derives mainly from their ability to simultaneously deliver a large dose to the tumour while mostly sparing surrounding healthy tissues ${ }^{10}$. Some researchers rightly caution that the existing knowledge and understanding of SMN induction is not sufficiently complete to justify the use of some advanced radiotherapies for treating children ${ }^{12}$. Much of the controversy is related to the carcinogenic risk from secondary neutrons, which are unavoidably present with particle treatments ${ }^{13}$. Quantifying this risk requires detailed knowledge of an extensive array of parameters. These dose and risk assessments are complex, expensive and require a multidisciplinary research team ${ }^{14}$.

The objective of this Review is to discuss recent advances in our understanding of the effect of advanced radiotherapies on the risk of SMNs in survivors of childhood cancer.

\section{Radiotherapy for paediatric patients}

Detailed statistics on the risk of SMNs related to radiotherapy are difficult to obtain because cancer in children is relatively rare. In children, lymphoma, leukaemia, brain tumours, sarcomas, Wilm's tumour, neuroblastoma and liver cancers are typically treated with radiotherapy ${ }^{15}$. Paediatric patients with tumours in the central nervous system, skull base and bone are those expected to benefit most from treatment with protons ${ }^{16}$ or carbon ions ${ }^{17}$. Even though the number of patients treated is fairly small, data obtained so far suggest that proton beam therapy can achieve better local control with no increase in acute toxicity for many childhood solid tumours ${ }^{18}$.

Eric J. Hall ${ }^{12}$ addressed three major factors that contribute to an increased risk of SMNs in children. First, the atomic bomb survivors database clearly shows that cancer risk depends on the age at exposure and decreases from about $15 \%$ per unit dose equivalent $\left(\mathrm{Sv}^{-1}\right)$ at under 10 years of age to about $1 \% \mathrm{~Sv}^{-1}$ for adults exposed at over 60 years age (see BOX 1 
for the definition of dose and $\mathrm{Sv}$ ). Second, the problem of genetic susceptibility is more serious for children than for adults, in whom carcinogenesis is often associated with lifestyle choices and environmental factors. Many cases of childhood cancer involve a germline mutation, such as the hereditary form of retinoblastoma, and this raises the question of whether the paediatric patient is also more sensitive and susceptible to radiation-induced cancer. Finally, the issue of stray radiation is more serious for children than for adults, simply because of the difference in body size ${ }^{12}$.

In its most recent report focused on SMNs, the Childhood Cancer Survivor Study (CCSS) screened 14,359 survivors, and found 2,703 neoplasms ${ }^{1}$. Survivors of Hodgkin's lymphoma and Ewing's sarcoma were those at highest SMN risk. A relationship between the relative risk and the dose received for the treatment of the primary cancer could be established for SMNs in the central nervous system, breast and thyroid. Gliomas generally occurred in the high-dose region before an attained age of 30 years in patients who had previously been treated for brain tumours, while breast cancers were observed in low-dose regions after the patients were older than 30 years of age. Taken together, the dose-response relationships, the relative risks and the latency periods for SMNs in the CCSS are consistent with what was expected from the large database of atomic bomb survivors ${ }^{19}$.

\section{Novel techniques in radiotherapy}

In the past few years, enormous progress has been made in radiotherapy ${ }^{20-22}$, leading to the possibility of providing escalating radiation doses (BOX 1) to the tumour while sparing the normal surrounding tissue (FIG. 1). This rapid progress has been boosted by advances in imaging tools to identify the tumour, in computerized treatment planning systems and in the development of radiation treatment machines.

Contemporary radiotherapy techniques often produce photoneutrons or use accelerated charged particles. Therefore, one of the most important issues is the influence of radiation quality on carcinogenesis. The difference between sparsely and densely ionizing radiation is in the initial energy deposition patterns. The relative biological effectiveness (RBE) of a given radiation is related to the spatial density of energy deposition events, known as the linear energy transfer (LET). A dose delivered by photons (sparsely ionizing or low-LET radiation) is more uniformly distributed than an equal dose of heavy ions (densely ionizing or high-LET radiation). Protons at high energy are similar to X-rays, but at low energy their LET increases and their RBE is higher than a-particles at the same LET $^{23}$. These lowenergy secondary protons are responsible for the high RBE of neutrons, arguably the most effective particles in inducing late effects ${ }^{24}$.

For heavy ion therapy, target tissues are exposed to carbon ions, and distal organs to neutrons and scattered protons ${ }^{10}$.

\section{Photon therapy}

Because of the unfavourable depth-dose distribution of X-rays, more than one photon beam has to be used to increase the dose to the tumour while sparing the normal surrounding tissue ${ }^{20}$. The improvements in computerized tomography (CT) imaging in the 1980s made it 
possible to switch to CT-based radiotherapy and three-dimension conformal radiotherapy (3D-CRT). The ability to modulate the intensity of the different beams - intensitymodulated radiation therapy (IMRT) - yielded better target to normal tissue dose ratios (in the moderate-high dose region) and improved sparing of important structures compared with 3D-CRT ${ }^{25,26}$. When combined with image-guided radiotherapy (IGRT) technologies, which take into account daily organ motions and tumour shrinkage, IMRT is the 'state of the art' photon therapy ${ }^{27}$.

However, as first pointed out by Hall and $\mathrm{Wuu}^{28}$, the move from 3D-CRT to IMRT has a cost: the risk of SMNs can increase, and may in fact be almost twofold higher. This is essentially caused by two problems: IMRT requires many more fields from different angles than conformal therapy, and therefore a larger volume of normal tissue is exposed to radiation; the exposure of out-of-field tissues from leakage $\mathrm{X}$-rays is also increased, because IMRT requires twofold to threefold more monitor units to deliver a specified dose to the target compared with 3D-CRT.

\section{Particle therapy}

In parallel to advances in photon therapy, radiotherapy with charged particles has evolved substantially since it was originally proposed more than 60 years ago $^{29}$. The rationale of particle therapy is simple: unlike photons, charged particles deposit most of their energy near the end of their range, in the region of the so-called Bragg peak. The Bragg peak is spread out to cover the whole tumour volume, and the exit dose - the dose beyond the tumour - is lower than with photon therapies. Particle therapy can therefore provide excellent dose conformation to the target while simultaneously providing better sparing of normal tissues ${ }^{10}$ (FIG. 1d). Heavy ions (such as carbon) provide physical advantages compared with photon beams and have a radiobiological benefit. Although high-energy protons have similar LET, and therefore RBE, as photons, carbon ions have low-LET in the entrance region, where the normal tissue is exposed, but high-LET at the end of their range, where the tumour is located ${ }^{30}$. Heavy-ion therapy is therefore preferable for radioresistant cancers, such as sarcomas, renal cell carcinoma, melanoma and glioblastoma ${ }^{11}$. To treat deep-seated tumours with heavy ions, complex treatment systems are required. The size and cost of such systems are presently larger than those of photon therapy; new technologies and economies of scale are expected to lead to smaller, less expensive systems within the next few years.

Currently, more than 20 centres are actively treating patients, and over 70,000 cancer patients have received particle therapy ${ }^{29}$. Most of these patients were treated with protons, but the use of heavier ions is increasing ${ }^{10}$. The debate regarding the cost/benefit ratio of this treatment is still ongoing ${ }^{9}$, but many more centres are currently under construction and the number of cancer survivors, especially paediatric patients, treated with charged particles is rapidly increasing ${ }^{10}$. These facts increase the urgency to better understand the risks of SMNs: in fact, neutrons and heavy ions are more effective than photons in cancer induction $^{31}$. 


\section{Potential risks of new techniques}

In the past few years, the concerns and controversy about the risk of SMNs following particle therapy has reached unprecedented levels ${ }^{12,32}$. This controversy is related to the high cost of the particle therapy facility and the debate regarding the cost/benefit ratio ${ }^{9}$, but it is partly a consequence of a lack of reliable dose and risk assessments tools. Available radiotherapy treatment planning systems do not provide accurate out-of-field dose calculations far from the treatment target ${ }^{32-34}$. At present, only a few institutions have the capability to carry out whole-body dose and risk assessments for conventional and advanced radiotherapies ${ }^{32,35,36}$.

Leakage neutrons are a problem for both high-energy photon and charged-particle therapies (FIG. 2a). In fact, photons at energies above 8-10 MV (depending on the target material) produce neutrons by photonuclear reactions (FIG. 2b). High-energy photons provide a better depth-dose distribution and are therefore preferred over lower energy X-rays for treating deep-seated tumours. High-energy photons interact with materials in collimators and the beam delivery system and can cause photonuclear reactions, which yields unwanted neutrons that contribute to the SMN risk. Photoneutrons are major contributors to doses of $18 \mathrm{MV}$ photons at distances $>20 \mathrm{~cm}$ from the target ${ }^{32}$, and the dose tends to flatten rather than decrease at greater distance. The patient is therefore immersed in a 'neutron bath' (FIGS 1b,2a).

With passive proton beam shaping, many neutrons are produced in the treatment unit ${ }^{32,37}$, and it has been argued that the leakage of these neutrons may substantially increase the risk of SMNs in distal organs ${ }^{13}$ (FIG. 2). Heavy ions also produce secondary particles by nuclear fragmentation, and these particles can deposit their energy in the normal tissue ${ }^{38,39}$. Neutrons undergo nuclear collisions with protons in water, generating additional charged particles that can ionize surrounding molecules. Exposures to leakage neutrons can be reduced by using magnetically scanned beams instead of passively scattered beams ${ }^{30,32,37}$.

The production of secondary neutrons in radiotherapy has been measured for different facilities (FIG. 2b). A recent review ${ }^{32}$ shows substantial inter-centre variability: this variation is attributed to many factors, including differences in the treatment units, experimental techniques and reporting methods. When heavy ions are considered, the neutron yield is caused, in part, by the fragmentation of the ${ }^{12} \mathrm{C}$ projectiles ${ }^{39}$, which is not relevant in proton therapy. However, this effect is compensated for by the reduced number of ${ }^{12} \mathrm{C}$ ions necessary to deliver a given dose: approximately $10^{10} \mathrm{C}$ ions per treatment, a factor of 100 fewer ions than with proton therapy ${ }^{30}$.

Such estimates are affected by very large uncertainties, depending on the specific treatment, site, quality factor and distal organ under consideration. A detailed set of dose measurements for a patient were carried out at GSI Helmholtz Center, Darmstadt, Germany, in 2008, when it was discovered that a young woman treated for a skull-base chordoma with ${ }^{12} \mathrm{C}$ ions was pregnant ${ }^{40}$. Neutron and photon doses in the uterus region were monitored during the treatment using both active and passive detectors. The dose in the pelvis for the full treatment was $82 \mu \mathrm{Sv}$, and neutrons contributed about $30 \%$ to the total dose. Considering the 
uncertainty, an upper limit of $0.2 \mathrm{mSv}$ for the uterine dose was estimated ${ }^{40}$. Both the mother and the child are healthy and do not have radiotherapy-related complications 3 years after treatment.

Taken together, these data suggest that particle therapy is typically not causing an increase in the dose to distal organs compared with high-energy IMRT. However, great care should be taken in comparing these values, which generally refer to the effective dose ${ }^{41,42}$ (BOX 1), a controversial radiological unit. Indeed, effective doses use tissue weighting factors that are estimated by several stochastic end points, and do not include any age- or genderdependence in cancer risk ${ }^{43}$. Especially for paediatric patients, the assumption that weighting factors are independent of age at exposure is tenuous. Cancer is a tissue-specific disease, and there is no evidence that the shape of the dose-response curve is the same for different organs. The International Commission on Radiological Protection (ICRP) indeed recognizes that the use of effective dose in medical applications may be inappropriate and it would be more useful to calculate the risks for specific age and gender groups, using absorbed or equivalent doses to organs and tissues and age-related risk factors ${ }^{44}$.

\section{Mechanisms of radiation-induced carcinogenesis}

Ionizing radiation has been recognized as a carcinogenic agent by the World Health Organization for many years ${ }^{45,46}$. Although early indications of radiation-induced cancers came from radiologists and other radiation workers, certainly the main epidemiological evidence of radiogenic carcinogenesis in humans and its dose-response relationship comes from the 1945 atomic bomb (A-bomb) survivor cohort ${ }^{47}$. Leukaemia and many solid cancers (especially lung, colon, breast and thyroid cancer) have been linked to radiation exposure ${ }^{46}$. The risks of developing a solid tumour after radiation exposure are reasonably well described by linear dose-response functions in the dose range from $0.2 \mathrm{~Sv}$ to $2 \mathrm{~Sv}$ (FIG. 3). However, epidemiology does not provide the necessary information for SMNs in radiotherapy patients, in which a small volume is exposed to high doses, and the rest of the body to low doses. The SMN risk is then calculated using models: usually, the linear-nothreshold (LNT) assumption is adopted at low doses ${ }^{48}$, whereas models taking into account competition between cell killing and transformation are used in the high dose region ${ }^{49,50}$. Physical doses are converted into dose equivalents using weighting factors at low doses or RBE values at high doses (BOX 1), and then the risk of cancer incidence or mortality is estimated by the product of the equivalent dose and organ-, age- and gender-specific risk coefficients, which have been mostly derived from A-bomb survivors. The models are affected by substantial uncertainties, which can only be reduced with a better understanding of the mechanism of radiogenic carcinogenesis ${ }^{31,48}$.

What is the molecular mechanism of radiation-induced cancer, especially of therapy-related cancers? Although we do not have a comprehensive answer, it is well known that cancer is a complex multistep process, and radiation can influence both initiation and promotion. DNA damage and repair, particularly double-strand breaks (DSBs), genomic instability and epigenetic mechanisms have key roles ${ }^{48}$. There is no evidence that mechanisms of therapyrelated cancers are different from those of sporadic cancers ${ }^{51}$, and therefore SMNs resulting from radiotherapy would just be part of the general problem of radiation-induced 
carcinogenesis. The relative cancer risks among Japanese A-bomb survivors are generally greater than those among comparable subsets in studies of medically exposed individuals ${ }^{46}$, probably because of the sterilization effects at high doses and the fractionation effects ${ }^{52}$. However, there are a few specific mechanistic issues that are particularly relevant for SMNs in radiotherapy patients: the problem of genetic susceptibility, the possible presence of different mechanisms at low and high doses, and the problems of split doses and radiation quality.

\section{Genetic susceptibility}

Apart from lifestyle factors (such as, smoking and a poor diet), genetic susceptibility is a major confounding factor in determining therapy-related cancers ${ }^{52}$. Can a genetic mutation or polymorphism that is associated with the primary cancer affect the risk and the mechanism of radiation-induced carcinogenesis? Mutations in high-frequency but lowpenetrance genes, as well as in low-frequency but high-penetrance genes, can enhance predisposition to radiation-induced cancers. The large increased risk of contralateral second primary breast tumours in women carrying mutations in the high-penetrance genes BRCAI and $B R C A 2$ provides evidence that mutations in these genes are involved in both the initial cancer and the increased risk of development of the second breast cancer ${ }^{53}$. The other wellknown case is ataxia telangiectasia, in which the key gene ataxia telangiectasia mutated $(A T M)$ is also an essential signalling molecule for DNA DSB repair following exposure to radiation, and its mutation is linked to genomic instability and cancer ${ }^{54}$.

How many genetic defects that are associated with the initial cancer can predispose to a second, radiation-induced malignancy? The recently established Radiogenomics Consortium ${ }^{5}$ will carry out genome-wide studies to identify genes that are involved in late effects after exposure to radiation. This is certainly an ambitious programme, especially as a single nucleotide polymorphism (SNP) could be responsible for increased sensitivity. For patients with bilateral retinoblastoma, osteosarcoma is the most frequent SMN that is induced by radiotherapy, which suggests that heterozygosity for the tumour suppressor gene retinoblastoma $1(R B 1)$ may predispose to radiation-induced bone sarcomas. In fact, genetic mapping in mice showed that multiple loci confer a genetic susceptibility to a-particleinduced osteosarcoma ${ }^{55}$, and $R b 1$-heterozygous mice have an increased risk of a-particleinduced osteosarcoma ${ }^{56}$. Interestingly, Ink4a (an upstream regulator of RB1)-heterozygous mice did not have increased susceptibility but had reduced tumour latency after radiation exposure $^{56}$. Animal models and the whole-genome screening in radiotherapy patients are likely to increase our knowledge of the role of genetic predisposition to SMNs.

\section{Low doses versus high doses}

One special characteristic of SMNs following radiotherapy is that they can arise either in the irradiation field (high-dose region) or in the distal organs (low-dose region). The question is whether different biological mechanisms may be causing low dose- and high dose-induced carcinogenesis. The rationale behind this question is that radiation can affect both the initiation and the promotion steps in carcinogenesis. 
In tissues exposed to low doses, the LNT for the dose-response curve is based on the assumption that even a single electron can induce DNA DSBs that could result in an initial carcinogenic event, even though the probability of this happening is low ${ }^{57}$. At low doses, non-targeted effects ${ }^{58}$ may increase the cancer risk by increasing the size of the susceptible target from one single cell to a whole tissue or a part of the tissue, although a protective mechanism has also been hypothesized, in which damaged cells are removed from the organism by intercellular signalling to protect tissue stability.

In the high-dose region, ionizing radiation will effectively kill cells in the field. The resulting tissue inflammation can promote carcinogenesis ${ }^{59}$. The response of the microenvironment to radiation and/or oxidative stress is mediated by cytokines, including epidermal and fibroblast growth factors, interleukins and pro-inflammatory cytokines ${ }^{60}$. Activation of the cytokine transforming growth factor- $\beta$ (TGF $\beta$ ) is an early and persistent event in tissues that have been exposed to ionizing radiation, and it can have protective or damaging effects: for example, it can protect the stem cell compartment in the intestine, but it can also promote invasion and metastasis in the epithelial tissue ${ }^{61}$. Although epidemiological data clearly show that radiation-induced cancer risk is much higher for exposure during childhood, a recent analysis of the A-bomb survivor cohort suggests that the decrease of cancer risk with age is not continuous for exposure in adulthood ${ }^{62}$. In middle age a number of dormant tumours might be present, so exposure to radiation could cause tumour promotion ${ }^{63}$. Initiation is likely to be the dominant process in the exposure of young people. Therefore, in radiotherapy for paediatric patients, the main concern would be the low-dose exposure of distal organs, whereas for adults high-dose-induced in-field SMNs can be expected owing to inflammation and promotion in pre-neoplastic tissue.

Finally, are out-of-field tumours caused by only low-dose, stray radiation? A bystander effect in vivo can be seen after exposure to high doses of radiation, known as an abscopal effect ${ }^{64}$. Recent data have shown an increased incidence of medulloblastoma in Patched 1 (Ptchl)-heterozygous mice after high-dose ( $3 \mathrm{~Gy}$ ) exposure of the lower body ${ }^{65}$, and apoptosis of unirradiated bone marrow cells can be induced in mice exposed to $4 \mathrm{~Gy} \gamma$ rays ${ }^{66}$. These high-dose, non-targeted effects are systemic effects, which are transmitted through the nervous, immune or vascular systems, and may be long-range and significant. If radiation at low doses (FIG. 3a) does not have an effect on - or even protect against cancer induction ${ }^{67}$, the non-targeted (abscopal) effect of these high target doses could be responsible for SMNs in distal organs.

\section{Radiation quality}

Radiation quality and its influence on the carcinogenic mechanism is one of the main issues in radiation protection. The radiation weighting factors $w_{\mathrm{R}}$, which are used to calculate the equivalent dose (BOX 1), are among the few parameters that are not based on epidemiology but on laboratory research studies.

So far, there is no evidence that radiation carcinogenesis is based on different mechanisms for different radiation qualities ${ }^{31}$. Although high-LET radiation induces complex damage measured both at the DNA ${ }^{68,69}$ and the chromosomal ${ }^{70}$ levels, complex DNA lesions can also be induced by low-LET electrons and are often considered the crucial lesions that lead 
to late cellular consequences. Non-targeted effects are particularly important in the case of low-fluence high-LET (that is, a low number of particles each delivering a relatively high dose) radiation ${ }^{71}$, but as noted above it is still unclear how much non-targeted effects contribute to radiation carcinogenesis. Gene expression profiles are often very different following exposure to different radiation qualities ${ }^{72}$, and the same is observed switching from low doses to high doses ${ }^{73}$, but gene expression following radiation or any other mutagenic stressor is a complex, time-dependent phenomenon, and difficult to use as a biomarker of late end points such as SMNs.

\section{Protons}

For estimating cancer risk from protons, we must rely entirely on animal and cellular experiments. The US Air Force and NASA carried out one such study from 1963 to 1969 (REF. 74). About 2,000 rhesus monkeys and 5,000 mice were irradiated with protons of energies ranging from $32 \mathrm{MeV}$ to 2,300 $\mathrm{MeV}$ obtained using cyclotrons at various institutions. Exposures to electrons and X-rays were also carried out to enable the comparison of the effects of radiation of different qualities. The RBE for protons for acute mortality was about 1.0 to 1.1 , which is the RBE generally used in human therapy ${ }^{75}$. The dose-response relationships that could be derived were curvilinear and consistent with those found in other experimental studies of the effects of low-LET radiation. In female monkeys, endometriosis was a major radiation effect that contributed to a shortened lifespan ${ }^{74}$. Another notable finding was the increased incidence of malignant brain tumours in the monkeys exposed to $55 \mathrm{MeV}$ protons. In rodent systems, $250 \mathrm{MeV}$ protons have been shown to be slightly more effective than $\gamma$-rays for the induction of Harderian gland ${ }^{76}$ or mammary ${ }^{77}$ tumours. However, these data are insufficient to provide an estimate of the RBE for cancer induction at the different proton energies.

\section{Neutrons}

The maximum RBE values for neutrons are observed at low-dose rates, especially for those end points for which a pronounced sparing effect is observed with low-LET radiation. The estimates of neutron relative effectiveness are mostly based on animal studies (FIG. 3b), although A-bomb survivors were also exposed to neutrons. In an extensive series of studies at the French Alternative Energies and Atomic Energy Commission (CEA) on male rats, a fission-neutron dose of $20 \mathrm{mGy}$ was found to be equivalent to $1 \mathrm{~Gy}$ of acute $\gamma$-rays to induce both lethal and non-lethal tumours ${ }^{78}$. Another large database is available from the JANUS programme at the Argonne National Laboratory. In approximately 32,000 mice with acute and fractionated exposures to $\gamma$-rays or neutrons ${ }^{79}, \mathrm{RBE}$ ranged from 2 to 50 and increased with dose fractionation. In these mice, tumours of epithelial tissue were induced by the highest RBE values and tumours of connective tissue origin were induced by the lowest RBE values. Limited data from monkeys on the effects of high-dose total body irradiation showed that the relative risk (that is, the cancer mortality rate relative to mortality rate in unirradiated controls) was approximately 8 for the $\mathrm{X}$-irradiated group and 14 for the neutron-irradiated group ${ }^{80}$.

Although RBE for fission-spectrum neutrons has been measured in many experimental systems, less data are available for high-energy neutrons, which are produced in particle 
therapy ${ }^{30}$ (FIG. 2b) and in spacecraft ${ }^{31}$. A recent experiment in Medaka fish embryos exposed to a high-energy neutron source at the Los Alamos Neutron Science Center reported an RBE ranging between 25 and 48 relative to $\gamma$-rays for apoptosis in cells of the developing brain $^{81}$.

\section{Heavy ions}

Finally, for heavy ions we have probably the highest uncertainty in terms of predicting late effects in humans ${ }^{31}$. Although it is generally assumed that the RBE increases with LET values around 100-200 $\mathrm{keV}$ per $\mu \mathrm{m}$ and then decreases at higher values, it depends on several factors, including the tumour type. The RBE of $1 \mathrm{GeV}$ per nucleon ${ }^{56} \mathrm{Fe}$ ions for inducing cancer in mice was around 1 for leukaemia (FIG. 3b) but more than 40 for hepatocellular carcinoma ${ }^{82}$. How can the RBE be so different? The reason is probably due to the different nature of haematological and solid cancers. For haematological cancers radiation could act as an initiator, but for solid cancers (especially those strongly linked to inflammation, such as liver cancer) it could act mainly as a promoter. Although heavy ions are more effective than X-rays in the induction of chromosomal rearrangements, most of the aberrations are lethal, and the RBE for chromosome aberrations drops to about 1 in the surviving cell population ${ }^{70}$. Conversely, heavy ions are effective in the induction of inflammation ${ }^{83}$. In general, only the organs in the beam path are exposed to heavy ions, while distal organs receive neutrons and scattered protons, indicating that SMNs in heavyion therapy could be most relevant to adult patients.

\section{Fractionation}

A further complication in radiotherapy is caused by an incomplete knowledge of the biological effects of dose fractionation. In classical radiobiology textbooks it is stated that the same dose delivered at a low dose rate or in daily fractions is less effective than acute exposure (sparing effect), but the extrapolation from high- to low-dose rate is one of the main sources of uncertainty in radiogenic cancer risk estimates ${ }^{84}$. In a recent, careful review of available experimental data (including human, animal and in vitro data) on radiation carcinogenesis, Suit et al. ${ }^{85}$ concluded that fractionation does not necessarily lead to a reduced cancer risk. As some SMNs could be caused by exposure to low-dose neutrons, it should also be noted that some in vitro data suggested that an inverse dose-rate effect exists for neutrons - carcinogenesis would be enhanced at a low-dose rate compared with acute exposure to fission-spectrum neutrons ${ }^{86}$. The inverse dose-rate effect is controversial, it has not been confirmed in subsequent experiments, and it might be visible and relevant only in a small window of dose rates, doses and $\mathrm{LET}^{87}$. The issue of carcinogenicity of split doses also remains unclear both in high- and low-dose regions, and more experiments to clarify this issue are urgently needed.

\section{Estimates of radiotherapy-related risk of SMNs}

Contemporary SMN risk models typically take into account various host-related factors that influence predicted risk, including age at exposure, attained age and sex ${ }^{62}$. Typically, risks are larger for females, decrease with age at exposure and continue to increase with attained age decades after exposure ${ }^{88}$. The subpopulation of patients who are at the greatest risk for 
the development of SMNs would potentially benefit the most by adding modifying factors to the risk models to take into account lifestyle choices, occupational exposures and genetic variation.

To accurately predict radiogenic risk using the models described above, the radiation doses in the tumour, as well as in healthy tissues and organs throughout the entire body, must be calculated. Fortunately, this is now possible, thanks to strident progress in radiation transport models, nuclear interaction data and models, and high-performance computing ${ }^{14}$. This means that, in principle, we can now calculate the dose to the patient and transform it to a cancer-risk map in the body (FIG. 4). In fact, our understanding of the physics of particle therapy is advanced and nearly complete for the purposes of SMN risk assessment. Most of the uncertainty remains in the biology (TABLE 1): we can calculate an organ dose fairly accurately, but the translation of the physical dose into an equivalent dose (BOX 1) and then to an organ-specific risk for cancer incidence and mortality is affected by large uncertainties that are caused by the uncertainties in weighting factors and cancer risk factors. The risk estimates in FIG. 4 are therefore only qualitative, but the calculation with the colour scale clearly shows how the dose is a poor predictor of risk, and large organ-specific differences can be found between incidence and mortality risks.

Studies comparing SMN risks that are associated with contemporary proton and photon therapies have consistently found that proton therapy confers smaller overall predicted risk of SMNs for children with medulloblastoma ${ }^{89,90}$ and for adults with prostate cancer ${ }^{91}$ and liver cancer ${ }^{92}$. The models found a negligible difference between lifetime risk of SMN incidence from passive versus scanned proton craniospinal irradiation: the risk was mostly attributable to therapeutic radiation, not leakage neutrons ${ }^{93-95}$. Similar findings were obtained for several patients treated for prostate cancer 91,96 . Perhaps the most important findings, however, are from comparative studies of passively scattered proton therapy and photon IMRT. These studies revealed that the largest reductions in predicted risk were obtained by replacing IMRT with proton therapy ${ }^{91}$, even after taking into account the stray and leakage neutrons that are associated with proton therapy. In fact, these findings were significant even when taking into account the large uncertainties in neutron RBE values (FIG. 2c) for carcinogenesis 90 .

\section{New challenges}

The problem of SMNs in paediatric radiotherapy patients is technically complex and challenging. There is good epidemiological evidence that radiation therapy is making a crucial contribution to long-term survival of childhood cancers, but it is also causing a high incidence of SMNs among survivors of childhood cancer. Given the size and consequences of this problem, it is vital that we meet our obligation to ensure that any avoidable and detrimental exposures to radiation are as low as can be reasonably achievable. However, it will be challenging to precisely define a reasonably low level of exposure in terms of risk/ benefit, and difficult to predict how much can be reasonably achieved with finite effort and expense $^{97}$. 
Fortunately, there are several avenues of research available to reduce SMN risks from particle radiotherapy. Modifying treatment units to reduce stray radiation is an obvious and attractive approach ${ }^{32}$. This can be accomplished by reducing the production of stray radiation inside the treatment unit and by increasing its attenuation with additional local shielding. For example, simple enhancements to a passively scattered proton nozzle (shielding) and treatment technique (distance) reduced the dose to paediatric patients by up to $40-50 \%{ }^{95}$. New research studies are needed to strengthen the initial evidence used to design personalized treatment strategies, such as the selection of radiation type that is likely to have the least detrimental effects, and treatment techniques, such as beam angles. The long latency times for SMNs require that research uses computational approaches to develop risk mitigation strategies, supplemented with radiobiological and radioepidemiological data as they become available.

Moreover, we should compare the stray therapeutic radiation with the dose from diagnostic procedures, which is rapidly increasing and causing concern for long-term risk in the population ${ }^{98,99}$. In most cases imaging during therapy accounts for less than $20 \%$ of the stray therapeutic radiation, but with the extensive applications of IGRT with daily portal imaging or cone-beam CT using MV X-rays, whole-body exposures of up to $100 \mathrm{mGy}$ per day are possible, thus exceeding those caused by the scatter radiation from the therapeutic beam ${ }^{100}$. When possible, orthogonal pairs of images with $\mathrm{kV} \mathrm{X}$-rays should be used to reduce the dose, considering that for paediatric patients the dose to important structures from $\mathrm{kV}$ cone-beam CT can be twofold to threefold higher than for adults ${ }^{101}$

The main research issues for SMNs in distal organs and for those in the radiation field for paediatric patients are summarized in TABLE 1 . In both high- and low-dose regions, the shape of the dose-response curve and its dependence on fractionation for carcinogenesis should be determined: this is clearly organ-specific, and it is therefore important to collect information about organ doses received during therapy. Even that could not be enough, as in some cases only a part of an organ is exposed to a high dose, and the other part to a low dose. How to deal with such a non-uniform exposure remains an open problem for late effects.

Despite the large uncertainties, data collected so far suggest that particle therapy should lead to a lower risk of SMNs than conventional X-ray techniques ${ }^{102}$. This is mainly attributed to lower doses to healthy tissues from the therapeutic beam and to the relatively low risk associated with neutron exposures to the whole body, especially with the spot-scanning delivery method. In other words, less normal tissue is exposed and distal organs receive lower stray radiation. This should also apply to heavy ion therapy, in which the therapeutic dose to healthy tissues is again very low and neutron production even lower than for protons, as fewer particles are needed to achieve the same target dose ${ }^{30}$. In fact, a lower yield of chromosomal aberrations is measured in the peripheral blood lymphocytes of patients treated with $\mathrm{C}$ ions compared with X-rays for the same cancers ${ }^{103,104}$. Some concerns may arise in the moderate-high dose regions, in which particles can be more effective in inflammation and promotion, although this mechanism may be more relevant for adult patients than for paediatric patients. 


\section{Acknowledgments}

This work was supported in part by the US National Cancer Institute (awards 1R01CA131463-01A1) and by Northern Illinois University, USA, through a subcontract of the US Department of Defense (award

W81XWH-08-1-0205). Research on particle therapy at GSI is partially supported by EU FP7 (ALLEGRO project), ESA (IBER-project) and Beilstein Stiftung (NanoBiC program). The authors would like to thank K. B. Carnes for her assistance in preparing this manuscript.

\section{Glossary}

\section{Medulloblastoma}

\author{
Atomic bomb \\ survivors database
}

Stray radiation

Radiation quality

Relative biological
effectiveness

a-particles

\section{Intensity-modulated radiation therapy}

\section{Monitor units}

Bragg peak
Malignant primary brain tumour that originates in the cerebellum or posterior fossa.

Survivors of the atomic bombs dropped in 1945 in Japan have been followed for cancers for more than 60 years, and represent the main source of epidemiological data on radiogenic cancers.

Therapeutic beam radiation that is emitted through the accelerator housing and reaches the patient outside of the treatment volume.

Ionizing radiation includes many different qualities: high-energy electromagnetic radiation (such as X-rays), neutrons, electrons, protons and heavy ions. Their different biological effectiveness is scaled using weighting factors (BOX 1).

The ratio of the dose, $D_{R}$, of a reference radiation (typically $\gamma$-ray or X-rays) and Dt of a test radiation (for example, neutrons, protons and heavy ions) that produce the same biological effect. It depends on several factors including the dose, dose rate, biological end point, radiation test LET and tissue.

Helium nuclei emitted by some heavy elements by a natural radioactive process known as a-decay. They represent the highLET component of the natural radiation background for the general population, mostly caused by inhalation of Radon gas.

Currently the most advanced type of photon radiotherapy. Accurate conformation to the target tumour is achieved by increasing the intensity of the rays to the target, and reducing the intensity of the beams that cross sensitive structures. The resulting inhomogenous dose distribution in the single field is compensated by cold and hot spots in the beams coming from other directions.

A monitor unit (MU) is a measure of the linear accelerator (Linac) output. The dose to the target is calibrated with a detector (monitor), and therefore MUs correspond to a given dose to the tumour.

Region where charged particles release most of their energy in matter, before stopping. 


\author{
Passive proton beam \\ shaping \\ Magnetically \\ scanned beams
}

Linear-no-threshold

Sterilization effects

Fractionation

Ataxia telangectasia

Non-targeted effects

Bystander effect

Abscopal effect

Harderian gland

Sparing effect
A technique that spreads the Bragg peak, using attenuators and collimators.

Also known as spot scanning, a technique to deliver particle therapy. A small pencil beam is deflected by a magnet in two dimensions to cover a slice of the tumour, and the next slice is exposed by changing the energy in the accelerator. Unlike passive beam shaping, it does not require attenuators to modulate the Bragg peak, and therefore the production of neutrons outside the patient's body is negligible.

The model commonly adopted by the International Regulatory Agencies to extrapolate the radiation risk at low doses. It is assumed that the cancer risk is always directly proportional to the absorbed dose.

At high radiation doses, the cell killing (sterilization) overcomes the radiation transforming potential, and hence the neoplastic transformation per exposed cell decreases.

The therapeutic radiation dose is very high (up to 60-70 Gy), but is normally delivered in daily fractions of approximately 2 Gy for effective sparing of the normal tissue.

A rare and severe neurodegenerative disease (also known as Boder-Sedgwick or Louis-Bar syndrome). It is caused by a defect in ATM, which encodes a serine/threonine protein kinase involved in DNA repair and cell cycle regulation.

Radiation effect observed in cells, tissue or organs not directly exposed to radiation. It can be caused by cell-to-cell communication via gap junctions, release of cytokines in the body, or mediated by the immune or nervous system.

A non-targeted effect generally observed in cellular experiments. The 'bystander' cell can receive radiation damage, although only the neighbouring target cell is exposed.

A radiation response in an organ not directly exposed to the radiation field.

A subcutaneous accessory lacrimal gland found within the eye's orbit in many vertebrates (not in humans).

When the same radiation dose is delivered in multiple fractions, at intervals of several hours (typically 1 day), the biological damage is generally reduced. 


\section{Fission-spectrum neutrons}

Neutrons produced during the nuclear fission process, typically in nuclear reactors for energy production. The energy spectrum peaks at about $1 \mathrm{MeV}$.

\section{References}

1. Smith MA, et al. Outcomes for children and adolescents with cancer: challenges for the twenty-first century. J. Clin. Oncol. 2010; 28:2625-2634. [PubMed: 20404250]

2. Friedman DL, et al. Subsequent neoplasms in 5-year survivors of childhood cancer: the Childhood Cancer Survivor Study. J. Natl. Cancer Inst. 2010; 102:1083-1095. [PubMed: 20634481] These are the most recent results of the CCSS, the most important epidemiological analysis of SMNs in children.

3. Oeffinger KC, et al. for the Childhood Cancer Survivor Study. Chronic health conditions in adult survivors of childhood cancer. N. Eng. J. Med. 2006; 355:1572-1582.

4. Robison LL, et al. The Childhood Cancer Survivor Study: a National Cancer Institute-supported resource for outcome and intervention research. J. Clin. Oncol. 2009; 27:2308-2318. [PubMed: 19364948]

5. West C, Rosenstein BS. Establishment of a radiogenomics consortium. Radiother. Oncol. 2010; 94:117-118. [PubMed: 20074824]

6. Travis LB, et al. Cancer survivorship--genetic susceptibility and second primary cancers: research strategies and recommendations. J. Natl. Cancer Inst. 2006; 98:15-25. [PubMed: 16391368]

7. Tubiana M. Can. we reduce the incidence of second primary malignancies occurring after radiotherapy? A critical review. Radiother. Oncol. 2009; 91:4-15. [PubMed: 19201045]

8. Meropol NJ, Schulman KA. Cost of cancer care: issues and implications. J. Clin. Oncol. 2007; 25:180-186. [PubMed: 17210937]

9. Terasawa T, et al. Systematic review: charged-particle radiation therapy for cancer. Ann. Intern. Med. 2009; 151:556-565. [PubMed: 19755348]

10. Durante M, Loeffler JS. Charged particles in radiation oncology. Nature Rev. Clin. Oncol. 2010; 7:37-43. [PubMed: 19949433] A review of the open research topics in particle therapy, including both tumour control and the risk of complications.

11. Schulz-Ertner D, Tsujii H. Particle radiation therapy using proton and heavier ion beams. J. Clin. Oncol. 2007; 25:953-964. [PubMed: 17350944]

12. Hall EJ. Intensity-modulated radiation therapy, protons, and the risk of second cancers. Int. J. Radiat. Oncol. Biol. Phys. 2006; 65:1-7. [PubMed: 16618572] This paper started the heated debate on the risk of secondary cancers using IMRT and proton therapy.

13. Brenner DJ, Hall EJ. Secondary neutrons in clinical proton radiotherapy: a charged issue. Radiother. Oncol. 2008; 86:165-170. [PubMed: 18192046]

14. Newhauser WD. Complexity of advanced radiation therapy necessitates multidisciplinary inquiry into dose reconstruction and risk assessment. Phys. Med. Biol. 2010; 55:e1.

15. Reulen RC, et al. Long-term cause-specific mortality among survivors of childhood cancer. JAMA. 2010; 304:172-179. [PubMed: 20628130]

16. Yock TI, Tarbell NJ. Proton beam radiotherapy for treatment in pediatric brain tumors. Nature Clin. Pract. Oncol. 2004; 1:97-103. [PubMed: 16264827]

17. Combs SE, et al. Carbon ion radiotherapy for pediatric patients and young adults treated for tumors of the skull base. Cancer. 2009; 115:1348-1355. [PubMed: 19156905]

18. Merchant TE. Proton beam therapy in pediatric oncology. Cancer J. 2009; 15:298-305. [PubMed: 19672146]

19. Armstrong GT, Stovall M, Robison LL. Longterm effects of radiation exposure among adult survivors of childhood cancer: results from the Childhood Cancer Survivor Study. Radiat. Res. 2010; 174:840-850. [PubMed: 21128808] 
20. Lichter AS, Lawrence TS. Recent advances in radiation oncology. N. Engl. J. Med. 1995; 332:371-379. [PubMed: 7824000]

21. Bernier J, Hall EJ, Giaccia A. Radiation oncology: a century of achievements. Nature Rev. Cancer. 2004; 4:737-747. [PubMed: 15343280]

22. Bucci MK, Bevan A, Roach M 3rd. Advances in radiation therapy: conventional to 3D, to IMRT, to 4D, and beyond. CA Cancer J. Clin. 2005; 55:117-134. [PubMed: 15761080]

23. Goodhead DT, et al. Direct comparison between protons and alpha-particles of the same LET: I. Irradiation methods and inactivation of asynchronous V79, HeLa and C3H 10T1/2 cells. Int. J. Radiat. Biol. 1992; 61:611-624. [PubMed: 1349625]

24. Edwards AA. RBE of radiations in space and the implications for space travel. Phys. Med. 2001; 17:S147-S152.

25. Webb S. The physical basis of IMRT and inverse planning. Br. J. Radiol. 2003; 76:678-689. [PubMed: 14512327]

26. Bortfeld T. IMRT: a review and preview. Phys. Med. Biol. 2006; 51:R363-R379. [PubMed: 16790913]

27. Verellen D, et al. Innovations in image-guided radiotherapy. Nature Rev. Cancer. 2007; 7:949_ 960. [PubMed: 18034185]

28. Hall EJ, Wuu CS. Radiation-induced second cancers: the impact of 3D-CRT and IMRT. Int. J. Radiat. Oncol. Biol. Phys. 2003; 56:83-88. [PubMed: 12694826]

29. Halperin EC. Particle therapy and treatment of cancer. Lancet Oncol. 2006; 7:676-685. [PubMed: 16887485]

30. Schardt D, Elsässer T, Schulz-Ertner D. Heavy-ion tumor therapy: physical and radiobiological benefits. Rev. Mod. Phys. 2010; 82:383-425.

31. Durante M, Cucinotta FA. Heavy ion carcinogenesis and human space exploration. Nature Rev. Cancer. 2008; 8:465-472. [PubMed: 18451812]

32. Xu XG, Bednarz B, Paganetti H. A review of dosimetry studies on external-beam radiation treatment with respect to second cancer induction. Phys. Med. Biol. 2008; 53:193-241. A comprehensive review that includes a careful comparison of the secondary radiation from different radiotherapy facilities.

33. Krämer M, Durante M. Ion beam transport calculations and treatment plans in particle therapy. Eur. Phys. J. 2010; D60:195-202.

34. Howell RM, et al. Methodology for determining doses to in-field, out-of-field and partially in-field organs for late effects studies in photon radiotherapy. Phys. Med. Biol. 2010; 55:7009-7023. [PubMed: 21076193]

35. Stovall M, et al. Dose reconstruction for therapeutic and diagnostic radiation exposures: use in epidemiological studies. Radiat. Res. 2006; 166:141-157. [PubMed: 16808603]

36. Newhauser WD, et al. Can megavoltage computed tomography reduce proton range uncertainties in treatment plans for patients with large metal implants? Phys. Med. Biol. 2008; 53:2327-2344.

37. Schneider U. Secondary neutron dose during proton therapy using spot scanning Int. J. Radiat. Oncol. Biol. Phys. 2002; 53:244-251. et. al.

38. Gunzert-Marx K, et al. Secondary beam fragments produced by $200 \mathrm{MeVu}^{-1}{ }^{12} \mathrm{C}$ ions in water and their dose contributions in carbon ion radiotherapy. New J. Phys. 2008; 10:075003. A comprehensive set of measurements on neutrons produced by heavy ion therapy.

39. Yonai S, et al. Measurement of absorbed dose, quality factor, and dose equivalent in water phantom outside of the irradiation field in passive carbon-ion and proton radiotherapies. Med. Phys. 2010; 37:4046-4055. [PubMed: 20879566]

40. Münter M, et al. Heavy ion radiotherapy during pregnancy. Fertil. Steril. 2010; 94:2329, e5-7. [PubMed: 20493478] Direct evidence from a pregnant patient that particle therapy produced low levels of stray radiation in distal organs.

41. ICRU. Prescribing, recording, and reporting protonbeam therapy. ICRU Report No. 78. J. ICRU. 2007

42. Wambersie A, et al. The RBE issues in ion-beam therapy: conclusions of a joint IAEA/ICRU working group regarding quantities and units. Radiat. Prot. Dosim. 2006; 122:463-470. 
43. Brenner DJ. Effective dose: a flawed concept that could and should be replaced. Br. J. Radiol. 2008; 81:521-523. [PubMed: 18443016]

44. ICRP. Recommendations of the ICRP. Ann. ICRP. 2007:37. ICRP Publication 103.).

45. El Ghissassi F, et al. A review of human carcinogenspart D: radiation. Lancet Oncol. 2009; 10:751-752. [PubMed: 19655431]

46. UNSCEAR. Annex A: Epidemiological Studies of Radiation and Cancer. Vol. Volume I. United Nations Office; Vienna: 2006. Effects of Ionizing Radiation.

47. Pawel D, et al. Improved estimates of cancer sitespecific risks for A-bomb survivors. Radiat. Res. 2008; 169:87-98. [PubMed: 18159958]

48. Mullenders L, et al. Assessing cancer risks of lowdose radiation. Nature Rev. Cancer. 2009; 9:596604. [PubMed: 19629073]

49. Schneider U, Lomax A, Timmermann B. Second cancers in children treated with modern radiotherapy techniques. Radiother. Oncol. 2008; 89:135-140. [PubMed: 18707783]

50. Sachs RK, Brenner DJ. Solid tumor risks after high doses of ionizing radiation. Proc. Natl Acad. Sci. USA. 2005; 102:13040-13045. [PubMed: 16150705] An excellent model of risk at high doses, which is important for radiotherapy.

51. Allan JM, Travis LB. Mechanisms of therapy-related carcinogenesis. Nature Rev. Cancer. 2005; 5:943-955. [PubMed: 16294218]

52. Little MP. Cancer after exposure to radiation in the course of treatment for benign and malignant disease. Lancet Oncol. 2001; 2:212-220. [PubMed: 11905766]

53. Malone KK, et al. Population-based study of the risk of second primary contralateral breast cancer associated with carrying a mutation in BRCA1 or BRCA2. J. Clin. Oncol. 2010; 28:2404-2410. [PubMed: 20368571]

54. Negrini S, Gorgoulis VG, Halazonetis TD. Genomic instability-an evolving hallmark of cancer. Nature Rev. Mol. Cell. Biol. 2010; 11:220-228. [PubMed: 20177397]

55. Rosemann M, et al. Multilocus inheritance determines predisposition to a-radiation induced bone tumourigenesis in mice. Int. J. Cancer. 2006; 118:2132-2138. [PubMed: 16331598]

56. Gonzalez-Vasconcellos I, et al. Differential effects of genes of the $R b 1$ signalling pathway on osteosarcoma incidence and latency in a-particle irradiated mice. Radiat. Environ. Biophys. 2011; 50:135-141. [PubMed: 21063720]

57. Brenner DJ, et al. Cancer risks attributable to low doses of ionizing radiation: assessing what we really know. Proc. Natl Acad. Sci. USA. 2003; 100:13761-13766. [PubMed: 14610281]

58. Mothersill C, Seymour CB. Radiation-induced bystander effects-implications for cancer. Nature Rev. Cancer. 2004; 4:158-164. [PubMed: 14964312]

59. Coussens LM, Werb Z. Inflammation and cancer. Nature. 2002; 420:860-867. [PubMed: 12490959]

60. Barcellos-Hoff MH, Park C, Wright EG. Radiation and the microenvironment - tumorigenesis and therapy. Nature Rev. Cancer. 2005; 5:867-875. [PubMed: 16327765]

61. Ikushima H, Myazono K. TGF $\beta$ signalling: a complex web in cancer progression. Nature Rev. Cancer. 2010; 10:415-424. [PubMed: 20495575]

62. Shuryak I, Sachs RK, Brenner DJ. Cancer risks after radiation exposure in middle age. J. Natl. Cancer Inst. 2010; 102:1628-1636. [PubMed: 20975037] An important model of age-dependent cancer risk that suggests that promotion not initiation could be the most important factor in radiation carcinogenesis in adults.

63. Goss PE, Chambers AF. Does tumour dormancy offer a therapeutic target? Nature Rev. Cancer. 2010; 10:871-877. [PubMed: 21048784]

64. Prise KM, O'Sullivan JM. Radiation-induced bystander signalling in cancer therapy. Nature Rev. Cancer. 2009; 9:351-360. [PubMed: 19377507]

65. Mancuso M, et al. Oncogenic bystander radiation effects in Patched heterozygous mouse cerebellum. Proc. Natl Acad. Sci. USA. 2008; 105:12445-12450. [PubMed: 18711141] Evidence from an animal model that cancer in distal organs can be caused by a non-targeted (bystander) effect and not low dose, stray radiation. 
66. Burr KL, et al. Radiation-induced delayed bystander-type effects mediated by hemopoietic cells. Radiat. Res. 2010; 173:760-768. [PubMed: 20518655]

67. Calabrese EJ, Baldwin LA. Toxicology rethinks its central belief. Nature. 2003; 421:691-692. [PubMed: 12610596]

68. Dobbs TA, et al. Interplay of two major repair pathways in the processing of complex doublestrand DNA breaks. DNA Repair. 2008; 7:1372-1383. [PubMed: 18571480]

69. Jakob B, et al. Live cell microscopy analysis of radiation-induced DNA double-strand break motion. Proc. Natl Acad. Sci. USA. 2009; 106:3172-3177. [PubMed: 19221031]

70. Ritter S, Durante M. Heavy-ion induced chromosomal aberrations: a review. Mutat. Res. 2010; 701:38-46. [PubMed: 20398789]

71. Cucinotta FA, Durante M. Cancer risk from exposure to galactic cosmic rays: implications for space exploration by human beings. Lancet Oncol. 2006; 7:431-435. [PubMed: 16648048]

72. Ding LH, et al. Gene expression changes in normal human skin fibroblasts induced by HZEparticle radiation. Radiat. Res. 2005; 164:523-526. [PubMed: 16187761]

73. Ding LH, et al. Gene expression profiles of normal human fibroblasts after exposure to ionizing radiation: a comparative study of low and high doses. Radiat. Res. 2005; 164:17-26. [PubMed: 15966761]

74. Wood DH. Long-term mortality and cancer risk in irradiated rhesus monkeys. Radiat. Res. 1991; 126:132-140. [PubMed: 1850849]

75. Paganetti H, et al. Relative biological effectiveness (RBE) values for proton beam therapy. Int. J. Radiat. Oncol. Biol. Phys. 2002; 53:407-421. [PubMed: 12023146]

76. Alpen EL, et al. Tumorigenic potential of high-Z., high-LET charged particle radiations. Radiat. Res. 1993; 88:132-143. [PubMed: 7302123]

77. Dicello JF, et al. In vivo mammary tumourigenesis in the Sprague-Dawley rat and microdosimetric correlates. Phys. Med. Biol. 2004; 49:3817-3830. [PubMed: 15446807]

78. Wolf C, et al. Neutron RBE for induction of tumors with high lethality in Sprague-Dawley rats. Radiat. Res. 2000; 154:412-420. [PubMed: 11023605]

79. Grahn D, Lombard LS, Carnes BA. The comparative tumorigenic effects of fission neutrons and cobalt-60 gamma rays in the B6CF1 mouse. Radiat. Res. 1992; 129:19-36. [PubMed: 1728054]

80. Hollander CF, Zurcher C, Broerse JJ. Tumorigenesis in high-dose total body irradiated Rhesus Monkeys - a life span study. Toxicol. Pathol. 2003; 31:209-213. [PubMed: 12696581]

81. Kuhne WW, et al. Biological effects of high-energy neutrons measured in vivo using a vertebrate model. Radiat. Res. 2009; 172:473-480. [PubMed: 19772468]

82. Weil MM, et al. Incidence of acute myeloid leukemia and hepatocellular carcinoma in mice irradiated with $1 \mathrm{GeV} /$ nucleon ${ }^{56} \mathrm{Fe}$ ions. Radiat. Res. 2009; 172:213-219. [PubMed: 19630525]

83. Rola R, et al. Hippocampal neurogenesis and neuroinflammation after cranial irradiation with $56 \mathrm{Fe}$ particles. Radiat. Res. 2008; 169:626-632. [PubMed: 18494546]

84. NCRP. Uncertainties in fatal cancer risk estimates used in radiation protection. NCRP; Bethesda, USA: 1997. Report No. 126

85. Suit $\mathrm{H}$, et al. Secondary carcinogenesis in patients treated with radiation: a review of data on radiationinduced cancers in human, non-human primate, canine and rodent subjects. Radiat. Res. 2007; 167:12-42. [PubMed: 17214511]

86. Hill CK, et al. Fission-spectrum neutrons at reduced dose rates enhance neoplastic transformation. Nature. 1982; 298:67-69. [PubMed: 7088163]

87. Brenner DJ, Hall EJ. Commentary 2 to Cox and Little: radiation-induced oncogenic transformation: the interplay between dose, dose protraction, and radiation quality. Adv. Radiat. Biol. 1992; 16:167-179. [PubMed: 11537507]

88. National Research Council. Health Risks from Low Levels of Ionizing Radiation: BEIR VII Phase 2. National Academies Press; Washington, DC: 2006.

89. Miralbell R, et al. Potential reduction of the incidence of radiation-induced second cancers by using proton beams in the treatment of pediatric tumors. Int. J. Radiat. Oncol. Biol. Phys. 2002; 54:824-829. [PubMed: 12377335] The first model of secondary cancers in paediatric patients treated with protons, suggesting that risk should be lower than for X-rays. 
90. Newhauser WD, et al. The risk of developing a second cancer after receiving craniospinal proton irradiation. Phys. Med. Biol. 2009; 54:2277-2291. [PubMed: 19305036]

91. Fontenot JD, Lee AK, Newhauser WD. Risk of secondary malignant neoplasms from proton therapy and intensity-modulated X-ray therapy for early-stage prostate cancer. Int. J. Radiat. Oncol. Biol. Phys. 2009; 74:616-622. [PubMed: 19427561] A direct comparison of predicted SMN risk for IMRT and protons indicating that a lower risk is expected for particles.

92. Taddei PJ, et al. Risk of second malignant neoplasm following proton versus intensity-modulated photon radiotherapies for hepatocellular carcinoma. Phys. Med. Biol. 2010; 55:7055-7065. [PubMed: 21076199]

93. Taddei PJ, et al. Effective dose from stray radiation for a patient receiving proton therapy for liver cancer. AIP Conf. Proc. 2009; 1099:445-449. [PubMed: 20865142]

94. Taddei PJ, et al. Predicted risks of second malignant neoplasm incidence and mortality due to secondary neutrons in a girl and boy receiving proton craniospinal irradiation. Phys. Med. Biol. 2010; 55:7067-7080. [PubMed: 21076189]

95. Taddei PJ, et al. Reducing stray radiation dose for a pediatric patient receiving proton cranospinal irradiation. Nucl. Techn. 2009; 168:108-112. Evidence that simple counter measures can effectively reduce the secondary dose in paediatric patients.

96. Taddei PJ, et al. Reducing stray radiation dose to patients receiving passively scattered proton radiotherapy for prostate cancer. Phys. Med. Biol. 2008; 53:2131-2147. [PubMed: 18369278]

97. Ottolenghi A, Smyth V, Trott KR. The risk to healthy tissue from the use of existing and emerging techniques for radiation therapy. Radiat. Prot. Dosim. 2011; 143:533-535.

98. Brenner DJ. Medical imaging in the 21 st century — getting the best bang for the rad. N. Eng. J. Med. 2010; 362:943-945.

99. Brenner DJ, Hricak H. Radiation exposure from medical imaging: time to regulate? JAMA. 2010; 304:208-209. [PubMed: 20628137]

100. Green S, Aird E. Imaging in radiotherapy. Br. J. Radiol. 2007; 80:967-969. [PubMed: 18065643]

101. Deng J, et al. Kilovoltage imaging dose in the radiotherapy of pediatric patients. Int. J. Radiat. Oncol. Biol. Phys. Apr.2011 6 [e-pub ahead of print].

102. Chung CS, et al. Comparative analysis of secondary malignancy risk in patients treated with proton therapy versus conventional photon therapy. Int. J. Radiat. Oncol. Biol. Phys. 2008; 72:S8.

103. Durante M, et al. X-rays vs. carbon-ion tumor therapy: cytogenetic damage in lymphocytes. Int. J. Radiat. Oncol. Biol. Phys. 2000; 47:793-798. [PubMed: 10837966]

104. Hartel C, et al. Chromosomal aberrations in peripheral blood lymphocytes of prostate cancer patients treated with IMRT and carbon ions. Radiother. Oncol. 2010; 95:73-78. [PubMed: 19800142]

105. Mole, RH. Dose-response relationships. In: Boice, JD.; Fraumeni, JF., editors. Radiation Carcinogenesis: Epidemiology and Biologic Significance. Raven Press; New York: 1984. p. 263-271. 


\section{Box 1}

\section{Radiation dose}

\section{Absorbed dose}

Absorbed dose (or dose) is the energy deposited per unit mass in the target: $D=\Delta E / \Delta D$ This is measured in gray (Gy). $1 \mathrm{~Gy}=1 \mathrm{~J}$ per $\mathrm{kg}$.

\section{Equivalent dose}

The equivalent dose (or radiation-weighted dose) takes into account the different biological effectiveness of different radiation qualities. $H_{T}=\sum_{R} w_{R} D_{T, R}$, in which $D_{\mathrm{T}, \mathrm{R}}$ is the absorbed dose (in Gy) averaged over the tissue or organ (T) owing to radiation (R). $H$ is measured in sievert (Sv).

The weighting factors $\left(w_{\mathrm{R}}\right)$ are estimated by relative biological effectiveness (RBE) values at low doses and low dose rates, and they are therefore useful for radiation protection at low doses, but not for protection from deterministic effects at high doses. In this case, the International Commission on Radiological Protection (ICRP) recommends a different definition of the equivalent dose: $H_{e}=\sum_{R} R B E_{R, e} \cdot D_{R}$, in which the the weighting factors are replaced with RBE values, which are radiation $(R)$ - and end point (e)-specific. The unit is the gray-equivalent (GyE).

\section{Effective dose}

For non-uniform exposure to radiation, different tissue weighting factors $w_{T}$ have to be used that reflect the different radiogenic sensitivity of the different organs. The effective dose (also in Sv) is then calculated as: $H_{E}=\sum_{T} w_{T} H_{T}$

\section{RBE-weighted absorbed dose}

In particle therapy, owing to the use of high doses, the GyE has been used for heavy ions, while the proton therapy community still uses the cobalt gray equivalent (CGE), which refers to a dose that is clinically isoeffective to ${ }^{60} \mathrm{Co} \gamma$-rays, formerly the most commonly used radiation in teletherapy. The International Commission on Radiation Units and Measurements (ICRU) has recently proposed ${ }^{41}$ to replace this quantity with the RBEweighted absorbed dose defined as: $D_{\mathrm{RBE}, V}=R B E \cdot D_{V}$

The unit will be again the gray but written as $\mathrm{Gy}(\mathrm{RBE})$. The volume $V$ (for example, gross tumour volume or planning target volume) must be specified.

\section{Integral dose}

Also important for comparing different radiotherapy modalities is the concept of integral dose, defined as the mean energy deposited in the total irradiated volume of the patient (in $\mathrm{kg} \times \mathrm{Gy}$ ): $I D=m \cdot D$

\section{Isoeffective dose}

In an effort to unify the RBE-weighted absorbed dose and the biologically effective dose, a joint IAEA and ICRU commission ${ }^{42}$ proposed another quantity indicated as 
isoeffective dose and defined as $D_{\mathrm{isoE}}=W_{\mathrm{isoE}} \cdot D$, in which $D$ is the absorbed dose in Gy and $W_{\text {isoE }}$ an inclusive weighting factor that takes into account all factors that could influence the clinical effects: dose per fraction, overall time, radiation quality, biological system and effects. The reference treatment conditions for estimating $W_{\text {isoE }}$ are X-rays, 2 Gy per fraction and 5 daily fractions per week. As for $D_{\mathrm{RBE}}$, the applications of $D_{\text {isoE }}$ in heavy-ion therapy is problematic owing to the complex RBE field. 


\section{At a glance}

- The availability of high-energy beams of photons, protons and carbon ions has contributed to increases in tumour control and the sparing of normal tissues from acute radiation toxicity.

- Advances in cancer therapies for children have produced impressive prospects for long-term survival. Approximately $80 \%$ of children and adolescents treated for cancer survive more than 5 years, but roughly $73 \%$ of them develop treatment-related complications. The complication of perhaps greatest concern is the risk for developing a radiogenic second malignant neoplasm (SMN), which can develop years or decades after treatment.

- Although the patient receives a high dose of therapeutic radiation, which is focused at the diseased tissue, the entire body is exposed to comparatively low doses of unwanted radiation that are caused by radiation leaking from the treatment apparatus and by scattering of the therapeutic radiation within the body.

- Mechanisms of therapy-related cancers are similar to those of sporadic tumorigenesis, but the carcinogenic potential of low doses of photons is not completely understood, and the uncertainty is much higher for cancer that is induced by charged particles.

- Epidemiological studies have conclusively shown that some SMNs can develop in tissues that are located in-field (that is, in the path of the therapeutic beam) and out-of-field (outside the path of the therapeutic beam).

- Recent models predict that particle therapy lowers the risk of SMNs compared with contemporary photon therapies.

- Regardless of the type of radiation beams used, nascent approaches to personalized, risk-adapted radiotherapy seem to be likely to yield further reductions in risk from out-of-field exposures, and research in genetic susceptibility and radiobiology should help to identify biomarkers of long-term risk in cancer survivors. 


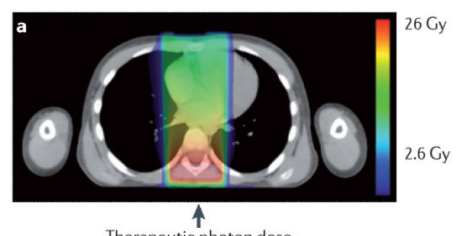

Therapeutic photon dose

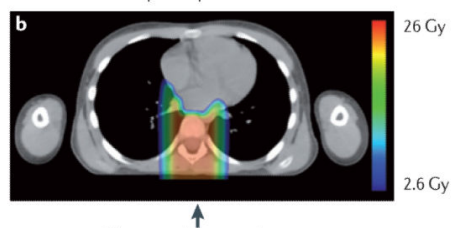

Therapeutic proton dose
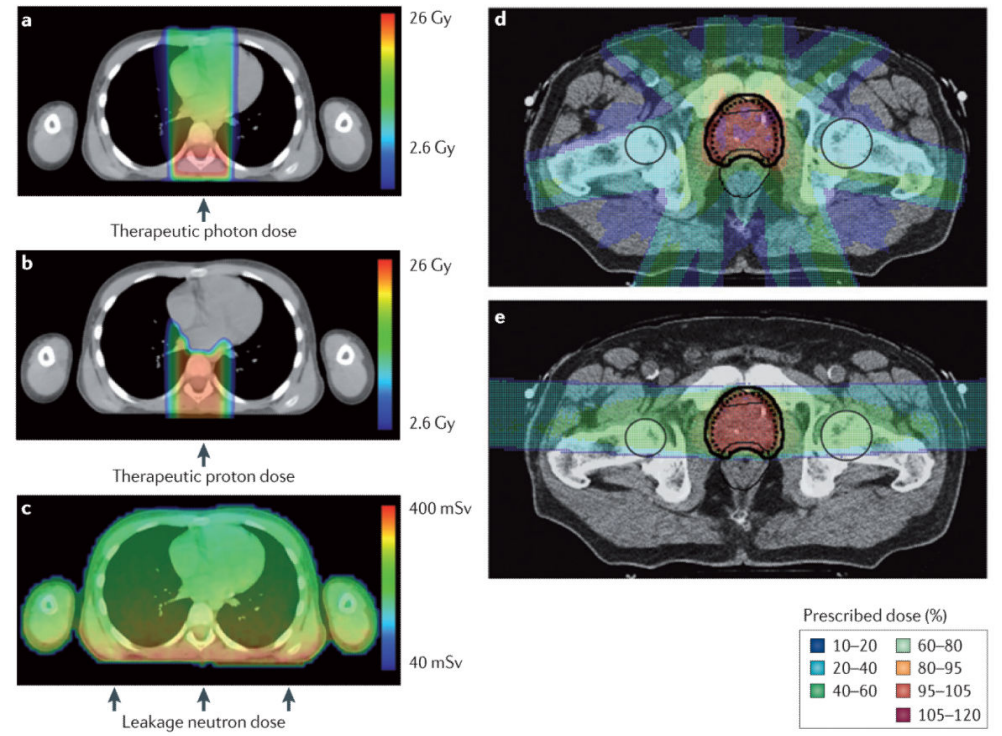

Prescribed dose $(\%)$

므 $10-20 \quad 60-80$

문-40 $\square 80-95$

무 $40-60 \quad$ 문 95

Figure 1. Treatment planning

Examples of treatment plans for craniospinal irradiation using photon (part a) or proton (parts $\mathbf{b}$ and $\mathbf{c}$ ) beams. The dose levels are shown as semi-transparent colour, superimposed on axial images of the patient from a computerized tomography scan. In the X-ray treatment (part a), the therapeutic radiation is highly localized laterally to the region of diseased tissue. Healthy tissues such as the heart and thyroid are in the beam path and receive large exit doses. For passive-scattered proton beams the therapeutic radiation (part b) is highly localized to the diseased tissues, whereas the comparatively low doses of leakage neutrons (part c) irradiate the entire body. Treatment plans for prostate radiotherapy are shown in parts $\mathbf{d}$ and $\mathbf{e}$, with intensity-modulated radiation therapy (part $\mathbf{d}$; seven coplanar fields) and a ${ }^{12} \mathrm{C}$ ion plan (part $\mathbf{e}$; two fields). Shown in greyscale is the computerized tomogram overlaid by the prescribed dose percentage in colour. The thick contour in black represents the clinical target volume, the dashed contour the gross tumour volume, and in thinner contours the rectum and the femoral head, which are tissues that are at risk from radiation. Parts $\mathbf{a}, \mathbf{b}$ and $\mathbf{c}$ are from the M.D. Anderson Cancer Center. Parts $\mathbf{d}$ and $\mathbf{e}$ are courtesy of A. Nikoghosyan and J. Debus, Heidelberg University Hospital, Germany. 


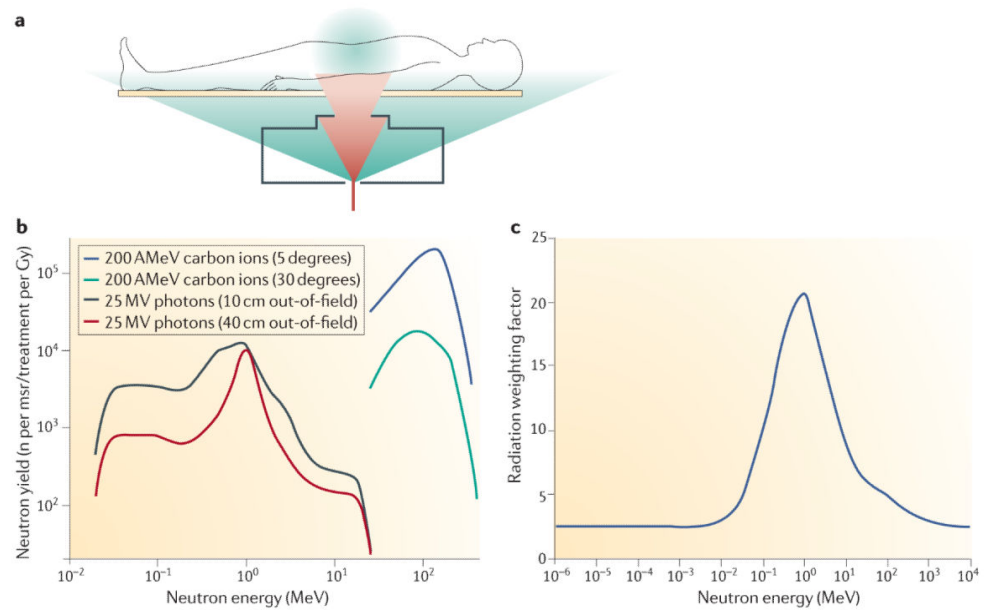

Figure 2. Secondary neutron dose in particle therapy

a Schematic diagram of a spinal treatment field in particle therapy. A small diameter beam of charged particles (red) enters the treatment apparatus, which spreads the beam to a clinically useful size and collimates it to spare healthy tissues. Stray neutron radiation (green) is created by proton-induced nuclear reactions in the treatment unit and in the patient. The neutron doses provide no therapeutic benefit but increase the predicted risk that a patient will develop a second cancer later in life as a result of radiation exposure. $\mathbf{b} \mid$ The energy spectrum of photoneutrons produced by megavoltage X-rays and secondary neutrons produced by nuclear interactions of charged particles is complex. The figure shows recent neutron spectral measurements at the ELEKTA Linac accelerator in the Klinikum Goethe Universität of Frankfurt, Germany, operated at $25 \mathrm{MV}$, and at GSI, Darmstadt, Germany, with a $200 \mathrm{MeV}$ per nucleon ${ }^{12} \mathrm{C}$ pencil beam stopping in a water target. The energy in $\mathrm{MeV}$ is on the $\mathrm{x}$ axis in log-scale, whereas the $\mathrm{y}$ axis gives the number of neutrons counted per unit solid angle (in millisteradiants (msr)) and per unit dose (in Gy) to the target.

Photoneutrons were measured at $10 \mathrm{~cm}$ or $40 \mathrm{~cm}$ from the target area. Secondary neutrons produced by the ${ }^{12} \mathrm{C}$ ions were measured at two angles from the beam path (for details of the measurements see REF. 38). The yield of neutrons decreases by increasing the distance from the target or the scattering angle, but clearly X-rays produce mostly neutrons around $1 \mathrm{MeV}$, and particle therapy neutrons with energies around $100 \mathrm{MeV}$. These different spectra result in different (organ-specific) risk factors. $\mathbf{c} \mid$ Neutron radiation weighting factor $w_{R}$ (BOX 1) is shown as a function of the neutron energy according to the latest International Commission of Radiological Protection recommendation ${ }^{44}$. The most effective neutrons are considered to be those with energies around $1 \mathrm{MeV}$. Part b courtesy of C. La Tessa, GSI, Darmstadt, Germany. 

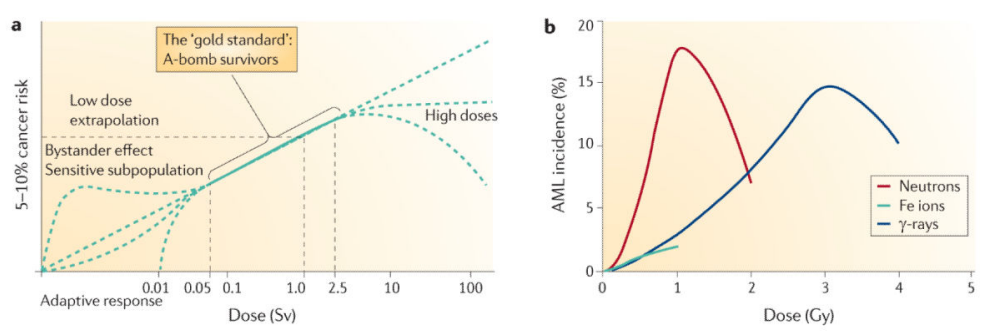

Figure 3. Dose-response curve for carcinogenesis

a Epidemiological data in humans are mostly derived from atomic bomb (A-bomb) survivors in the dose range 0.1-2.5 Sv. At lower doses, The International Commission on Radiological Protection (ICRP) ${ }^{44}$ recommends a linear extrapolation (the linear-nothreshold (LNT) model). However, the non-targeted (bystander) effect or the existence of radiosensitive subpopulations may suggest that the LNT model underestimates the risk ${ }^{58}$. Conversely, an adaptive response would imply that the LNT is overestimating the risk ${ }^{66}$. Ongoing research has not yet clarified the importance of these mechanisms in low-dose carcinogenesis ${ }^{48}$. Similarly, the extrapolation at high doses, relevant for the risk of in-field second malignant neoplasms (SMNs), is uncertain. The risk may decrease owing to cell killing (as suggested for second thyroid cancer in the Childhood Cancer Survivor Study (CCSS) study ${ }^{19}$ ), remain linear (as it seems for central nervous system SMNs in the CCSS database ${ }^{19}$ ) or plateau (as suggested by second lung and breast cancers in survivors of Hodgkin's lymphoma ${ }^{50}$ ). Cellular repopulation during and after the therapeutic radiation exposure may in fact counteract cell killing at high doses. $\mathbf{b} \mid$ An example of dose-response curves for the induction of cancer in animal models by radiation of different qualities. Acute myeloid leukaemia (AML) in CBA mice exposed to $\gamma$-rays, fission neutrons or $1 \mathrm{GeV}$ per nucleon ${ }^{56} \mathrm{Fe}$ ions. The relative biological effectiveness (RBE) is about 3 for neutrons and 1 for heavy ions. However, the RBE values depend on the genetic strain, tumour type and fractionation used. The curves represent guides drawn by eye from data points in REFS 82,105. Part a courtesy of E. J. Hall, Columbia University, USA. 

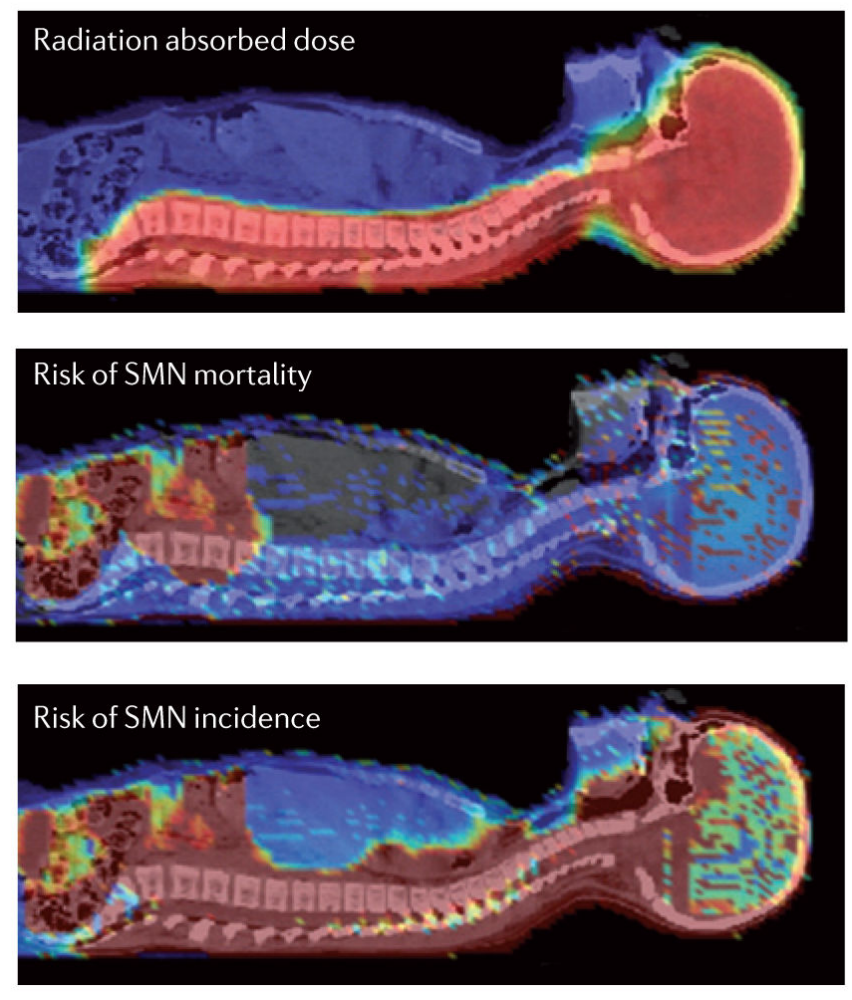

Figure 4. Dose and risk distribution for second cancer

A 9-year-old girl received craniospinal irradiation for medulloblastoma using passively scattered proton beams at the M.D. Anderson Cancer Center, USA. The colour scale illustrates the difference for absorbed dose, incidence and mortality cancer risk in different organs. Radiation absorbed dose depends strongly on patient anatomy and treatment factors. Risk of second malignant neoplasm (SMN) incidence and mortality varies strongly with radiation dose, but, importantly, it also varies strongly between organs, the age of the patient at exposure and the attained age, sex and genetic profile, as well as other factors.

Consequently, as this figure illustrates, dose is a poor biomarker for SMN risk. In future, novel risk visualization and analysis methods will be needed to facilitate routine riskadapted, personalized clinical decision making. 


\section{Table 1}

Key questions in paediatric SMN research using modern radiotherapy techniques

\begin{tabular}{lll}
\hline Main research problems & $\begin{array}{l}\text { In-field, moderate to high doses } \\
\text { of X-rays, protons or heavy ions }\end{array}$ & $\begin{array}{l}\text { Out-of-field, low doses of photons, } \\
\text { neutrons or scattered protons }\end{array}$ \\
Knowledge of organ and tissue doses & Accurate & $\begin{array}{l}\text { Large uncertainties, limited availability } \\
\text { of capability to calculate doses }\end{array}$ \\
$\begin{array}{l}\text { Organ-specific shape of the } \\
\text { dose-response curve for carcinogenesis }\end{array}$ & $\begin{array}{l}\text { X-rays and charged particles at } \\
\text { moderate to high doses }\end{array}$ & $\begin{array}{l}\text { Therapy-like neutron spectra and } \\
\text { mixed fields at low doses }\end{array}$ \\
Effect of fractionation & $\begin{array}{l}\text { Low- and high-LET radiation at high } \\
\text { doses }\end{array}$ & $\begin{array}{l}\text { Split doses of neutrons: inverse } \\
\text { dose-rate effect? }\end{array}$ \\
Genetic susceptibility & $\begin{array}{l}\text { For cancer promotion by } \\
\text { inflammation }\end{array}$ & For cancer induction (initiation) \\
Non-targeted effects & In tissue surrounding the PTV & $\begin{array}{l}\text { Abscopal or tissue-mediated effects in } \\
\text { distal organs }\end{array}$ \\
\hline
\end{tabular}

LET, linear energy transfer; PTV, planning target volume; SMN, second malignant neoplasm. 